\title{
Ein angiovinisches Gebetbuch in der Wiener Hofbibliothek.
}

\author{
Von \\ A. Riegl.
}

Wenngleich der Miniaturmalerei des späteren Mittelalters - namentlich der italienischen - nicht jene hervorragende Stellung in der Kunstgeschichte zukommt, die ihren Hervorbringungen in den vorangehenden Jahrhunderten eingeränmt werden muss, so verdient der Antheil, den die einzelnen Schulen an der reichen Entfaltung der Buchmalerei ihrer Zeit genommen haben, immerhin volle Beachtung. Vor allen sind es die Künstler der altsenesischen Schule vor den Lorenzetti, die durch ihre feine mühselige Technik auf dic Miniaturmalerei unmittelbar hingewiesen waren; es mag daher nicht zufällig sein, dass man mehrfache Denkmäler dieser Art mit dem Hauptmeister der Schule - Simone Martini - in Verbindung gebracht hat. Zur gleichen Zeit fand aber auch die durch Giotto in die Frescomalerei eingeführte breite malerische Behandlung durch florentinische Künstler in der Buchillustration Fingang. Für die Charakteristik beider Schulen bietet der Cod. Vind 1921 eine Reihe vortrefflicher Beispiele.

Der Handschriften-Catalog der Hof bibliothek weist den Codex einen Liber precum cum Calendario - dem 15. Jahrhundert zu und weiss bezüglich der Provenienz nur zu sagen, dass die Heiligennamen des Kalenders theils auf Frankreich, theils auf Schweden hindenten. Es ist dies in Kürze zusammengefasst das Resultat, zu dem Denis bei einer Beschreibung der Handschrift in den Codd. manuscripti bibliothecae palatinae Vindobonensis 2, $2173 \mathrm{n}^{0} 905$ gekommen war. Gegen diesen Zeitansatz sprechen jedoch sowol die Schrift, als der Charakter der Miniaturen. Die Handschrift ist nämlich zum allergrössten Theil, im wesentlichen bis fol. 327, von ein er Hand geschrieben und zwar unzweifelhaft von der Hand eines Italieners des 14. Jahrhunderts. 
Dies beweist sowol der Schriftcharakter, welcher durchaus der Scharfbrüchigkeit, wie sie der gothischen Schrift nordwärts der Alpen eigen ist, entbehrt; dies beweisen ferner zahlreiche sprachliche Eigenthümlichkeiten, wie die Worte istoria, pulcra, Margarita etc. Die letzten 20 Folien stammen wahrscheinlich von der Hand eines Franzosen und gehören auch noch dem 14. Jahrbundert an; vielleicht identisch damit ist die Hand, welche im Kalender die Namen zu den Bildern der einzelnen Thierkreiszeichen beigeschrieben hat. Ausserdem ist fol. 300 ausgefallen und von nicht viel späterer Hand eines Nichtitalieners ergänzt; endlich wurden im 15. Jahrhundert einzelne leergebliebene Seiten mit Gebeten in spätgothischer Schrift ausgefullt. Ebenso bestimmt weist uns auf den Süden und auf das 14. Jahrh. der Bilderschmuck. Abgesehen ron den letzten 20 Folien, deren übrigens spärliche Illuminirung ebenso wie die Schrift französischen Ursprungs sein dürfte, haben an der Ausschmückung der Handschrift mehrere Künstler theilgenommen, die sämmtlich den Charakter italienischer Malerei der ersten Hälfte des Trecento zur Schau tragen, wenn sie auch verschiedenen Schulen angehören. Mit diesem Zeitansatz stimmt auch die ormamentale Ausstattung überein. Es fehlen die selbständigen Randleisten der früheren und späteren Zeit; der Randschmuck in Gestalt von ornamentalem Geranke geht von den Initialen aus, die dafür auf den figürlichen Schmuck verzichten müssen, wodurch die Brücke zur folgenden Zeit, die das Vollbild selbständig ausbildet, geschlagen war.

Der Codex hat Octarformat und enthält ein Calendar, einen Psalter und verschiedene Gebete; seinen gegenwärtigen schweinsledernen Einband bekam er erst um das Jahr 1600 .

Das Calendar ist von einem giottesken Künstler illuminirt und zwar sowol mit Monatsbildern als auch mit bildlichen Darstellungen der Thierkreiszeichen. Beide weisen bemerkenswerthe Eigenthümlichkeiten auf.

Ganz abweichend von der im Norden seit dem 13. Jahrhundert üblichen Weise, die Kalenderbilder in Architecturen oder Medaillons hineinzucomponiren, befindet sich hier das Monatsbild am Anfange des jeweiligen Monats auf Goldgrund innerhalb eines viereckigen Rahmens, der auch das darunter befindliche $\mathrm{KL}$, das bekanntlich stets das Incipit einer Monatstafel bildet, auf blauem Grunde umschliesst. Das Thierkreiszeichen befindet sich dagegen am Ende der Monatstafel, gleichfalls in viereckigem Rahmen, auf blauem Grunde und weist die schon von Denis bemerkte Eigenthümlichkeit auf, dass die Thierbilder zu Darstellungen menschlicher Leiden verwerthet sind. Der 
Grundgedanke ist nämlich, eine nackte menschliche Gestalt von dem jeweiligen meist figürlich dargestellten Thierkreiszeichen peimigen zu lassen. Im Zeichen des Wassermannes erblicken wir den Menschen von grünen Wellen verschlungen, in folgenden wird er von den Fischen gebissen, vom Widder gestossen, vom Stier getreten usw. Das Männchen der Zwillinge durchbohrt ihn mit einem Dolche, der Schütze mit einem Pfeile, ja selbst die Jungfrau, eine schlanke Gestalt in langem violetten Kleide, fasst den Zusammenbrechenden heftig am Arme. Nur die Wage bot keine Handhabe für eine gleichartige Darstellung, und die rücklings niedergesunkene Gestalt des Menschen mit der Waage in der Hand erscheint völlig unmotivirt.

Aber auch die Monatsbilder bieten neben mancher Verwandtschaft mit den gleichzeitigen nordischen Darstellungen völlig fremde Züge. Während Frankreich und Deutschland mit geringen Abweichungen den gleichen Spiegel des Wechsels der Jahrzeiten in den menschlichen Bethätigungen darbieten, die in den einzelnen Monatsbildern vorgeführt werden, sehen wir uns hier in ein Land versetzt, wo man im Juni die reifen Aehren schneidet, im December säet. Der Monat, in dessen Bilde die meisten nordwärts der Alpen geschriebenen Kalender übereinstimmen - der December - , muss sein Schweineschlachten an den Februar abtreten ${ }^{\mathfrak{l}}$ ). Solche Abnormitäten passen weder für die Isle de France, noch für Süddeutschland, wol aber für die Campagna felice. - Die äusserst feine Ausführung in kleinen Verhältnissen eignet sich minder dazu, um an diesen Bildern die Eigenthümlichkeiten des Künstlers zu demonstriren: wir gehen daher zu den Vollbildern des Psalters über.

Der Psalter mit Canticis und Litanei reicht bis fol. 184 incl. und ist mit acht Bildern illuminirt, welche durchwegs von der Hand eines und desselben Meisters herrühren; nur mit Bezug anf das letzte (f. 131) könnte etwa ein Zweifel entstehen. Auf den ersten Blick glauben wir einen spätbyzantinischen Meister vor uns zu sehen: der wolstudirte regelmässige Faltenwurf, die faltigen Gesichter der Greise, der grünliche Ton des Fleisches sind zunächst geeignet, diesen Eindruck in uns zu erwecken. Doch beschränkt sich dieses byzantinische Element auf jene geringe Beimischung, die der altsenesischen Malerei überhaupt eigen ist. Die grünliche Untermalung der Fleischpartien ist durch die darüber gesetzten und sorgfaltig vertriebenen weissen und rothen Töne zu feiner Modellirung und Charakterisirung ver-

1) Nur die englischen bringen bisweilen ein Rinderschlachten. 
wendet'1); eines der glänzendsten Beispiele hiefür ist das Bild des; h. Ludwig von Toulouse (f. 223) [siehe Abb. 1], dessen jugendlichess Antlitz durch die Verschmelzung der drei Farben einen unendlich

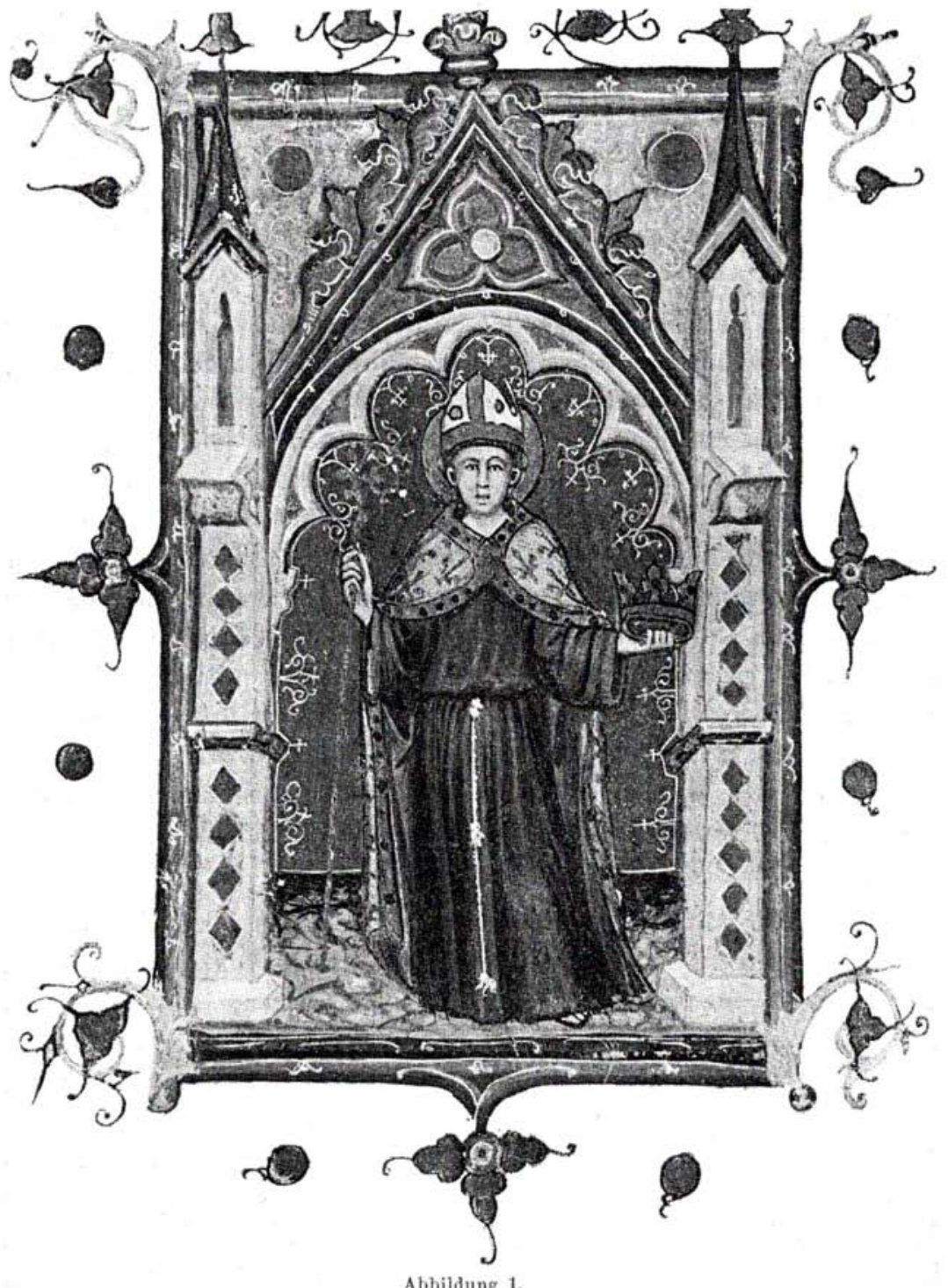

liebreizenden Ausdruck erhielt. Eine weitere charakteristische Eigenthümlichkeit, die diesen Seneser Meister einerseits in Zusammenhang

1) Vgl. die genaue Beschreibung der altsenesischen Maltechnik bei Crowe und Cavalcaselle, Ital. Mal. D. A. 2, $206 \mathrm{f}$. 
mit der älteren Kunstübung zeigt, andrerseits entschieden von den giottesken Künstlern scheidet, liegt in der reichlichen Anwendung von schwarzen Contouren. Nicht nur die äusseren Umrisse der Figuren, sondern theilweise auch die Vertheilung der Gewandmassen, ja die Modellirungen des Gesichtes sind mit schwarzen Tintenstrichen hervorgehoben, welche nach dem Auflegen deu Localfarben gemäss der Vorzeichnung nachgezogen wurden. Wo dies unterblieb, wie eben anf dem letzten Bilde des Psalters (f. 131), da wird es zum Verdachtsgrund gegen den gemeinsamen Ursprung. Es ist dieselbe Technik, die bis gegen 1350 in Frankreich üblich war, die Flächen zwischen den schwarzgezogenen Contouren mit den Localfarben auszufüllen. Von $1350 \mathrm{ab}$ tritt eine entschiedene Wendung zum Malerischen ein, die Abtonung der Farben gegen einander tritt an die Stelle der Umrissstriche, und darin sind, wie wir sehen werden, die giottesken Miniaturmaler den Franzosen bereits vorangegangen. Weniger eigenthümlich ist der senesische Meister in den Hintergründen und Architecturen: gerade in dem architectonischen Beiwerk italienisch-gothischen Stiles zeigt er sich mit dem giottesken Collegen einem Boden entwachsen; doch tritt beim Senesen, dem allgemeinen Charakter der Schule entsprechend, die Freude am Ornamentalen stärker hervor ${ }^{1}$ ). Die Köpfe der älteren Personen sind stark individualisirt, and charakteristisch erscheint eine Falte, die, von der Nase abwärts laufend, den Mund von den Wangen scheidet. In den jugendlichen Figuren dagegen. - namentlich in den weiblichen - offenbart sich ein vollendeter Liebreiz, wie er gleichfalls für die altsenesischen Meister charakteristisch ist?). Auch die Modellirung der Gewänder ist nicht mehr durchwegs an die Verwendung der Contouren gebunden, sondern vielfach malerisch behandelt, namentlich sind zarte Weisshöhangen reichlich angewendet.

Die Art und Weise, wie die Vollbilder in den Text hinein componirt sind, weist wol auf ihren Ursprung als ehemalige Füllungen von Initialen, aber durch ihre Beziehungen zum Inhalte erscheinen sie bereits als vollig selbständige Illustrationen des Textes.

Das 1. Bild (f. 13) zerfällt in zwei übereinander angeordnete Darstellungen, die aber ron einem gemeinsamen Vierpass umschlossen sind; letzterer ist ausserdem noch von einem Dornblattrahmen umgeben. Im oberen Bilde sehen wir König Saul den Speer zücken gegen den harfespielenden David. Ein Teufel - das ausgebildete nordische Phantom - ist beflissen, die zaudernde Rechte des Königs zu dem verderblichen Wurfe emporzuheben. Im unteren Bilde ge-

1) vgl. Crowe und Car. a. a. O.

?) vgl. Crowe und Cav. \&, 235. 
wahren wir den jungen David, wie er dem hingestreckten Riesen Goliath das Haupt abschlägt.

Das zweite Bild (f. 36) bringt die Salbung Davids: Samuel giesst aus einem Horne das Salböl über das Haupt des mit gefalteten Händen sitzenden Jünglings. Ein flacher Rundbogen umschliesst nach oben die Darstellung.

Am dritten Bilde (f. 51) kniet König David, die Rechte zum Schwur ausgestreckt, während der Zeigefinger der Linken den Mund berührt. Von rechts schweht die segnende Halbfigur des Erlösers heran, in weissem Gewande, mit Kreuznimbus, auf blauem mit feinen weissen Ornamenten verzierten Grunde, durch einen Regenbogen vou dem rautengemusterten Grunde geschieden, von dem sich die Gestalt des Königs abhebt. Es beginnt der Psalm 38, 2: Dixi custodiam vias meas, ut non delinquam in lingua mea, posui ori meo custodiam.

Auf dem vierteu Bilde (f. 65) gewahren wir wieder Christus ganz in derselben Weise heransohwebend, austatt des Königs aber einen halbnackten Thoren, einen Knüttel schwingend wider den Erlöser, während drei Hunde zähnefletschend heranspringen, den Frevler zu zerreissen. Es beginnt der Psalm 52, 1: Dixit insipiens in corde suo : non est deus...

Und noch einmal begegnen wir derselben Gestalt Christi auf dem fünften Bilde (f. 79), zur Linken aber wiederum König David, der in einem auf grünen Wogen tanzenden Schifflein sitzend die Hände gegen den Erlöser faltet. Das Bild zeugt von trefflicher Naturbeobachtung: es bezieht sich auf den Psalm 68, 2: Salrum me fac deus quoniam intraverunt aque usque ad animam meam ... veni in altitudinem maris et tempestas dimersit me.

Auf f. 96' gewahren wir König David sitzend vor einer Orgel, deren Blasebalg er tritt; gleichzeitig schlägt er mit 2 Hämmern an die 7 Glocken des über ihm hängenden Glockenspieles. Es beginnt der Psalm 80, 2: Exultate deo adjutori nostro, iubilate deo Jacob. Sumite psalmum et date tympanum, psalterium iocundum cum cythara, buccinate in neomenia et tuba. Den Rahmen bildet ein kreuzblumenbekrönter Kielbogen von Strebepfeilern flankirt.

Bas siebente Bild (f. 113) bringt die übliche Illustration zum Psalm 95, 1: Cantate domino canticum novum - singende Mönche mit trefflich individualisirten Köpfen, je 3 zu beiden Seiten eines Doppelpultes, auf dem die Notenbücher aufliegen. Die Architectur weist hier auf eine etwas frühere Stufe: eine dreischiffige Halle auf 2 Pilastern und 2 mittleren Säulchen ruhend, die Rundbogen der Seitenhallen niedriger, wogegen der gestelate Kleebogen in der Mitte, 
von dessen Scheitel eine Lampe herabhängt, höher hinansteigt. Die Giebelkrönung ist aber gothisch.

Das letzte Bild des Psalters (f. 131) zeigt Gott Vater und Gott Sohn auf einer Bank nebeneinander sitzend, von einer Kielbogenarchitectur eingeschlossen. Von oben schwebt der $h$. Geist als Taube herab. Der heraldisch zur Linken sitzende Vater (Psalm 109, 1: Dixit dominus domino meo : sede a dextris meis) hält die Weltkugel, der Sohn ein Buch. Beide haben ihre Rechte halbgeöffnet ron sich gestreckt. Es wurde schon früher bemerkt, dass auf diesem Bilde die schwarzen Umrisszüge fehlen; die Gewänder sind trefflich geworfen, der Kopf, namentlich Gott Vaters, streng und feierlich gebildet.

Mit f. $185^{\prime}$ beginnen die Preces matutinales. Gleichsam als Titelbild gewahren wir die Anbetung der Madonna mit dem Kinde durch eine knieende Königin. Diese ist in ein weitausgeschnittenes rothes Kleid gehüllt, die grünen Aermel haben die der Zeitmode eigenthümlichen weissen Hängeärmel. Um Hinterhaupt und Hals ist ein durchsichtiger Schleier gelegt, das Haupt mit der Lilienkrone geschmückt. Diese öfter wiederkehrende Figur wurcle, wie wir sehen werden, schon von Denis bemerkt und zu deuten gesucht. Hinter der Königin sind Dienerinnen sichtbar. Hier begegnen wir einem 'giottesken Meister. Die schwarzen Contouren verschwinden und an ihre Stelle tritt die Abtonung der Farben gegeneinander; die Köpfe werden lediglich durch mehr oder minder starke Röthe des Fleischtons modellirt. In den Draperien tritt eine Vorliebe für gelbe Gewänder mit rothen Brüchen hervor, ebenso die Neigung zu breiten rundlichen Parallelfalten im Gegensatze zu dem zierlichen Gefältel der byzantinischen Weise. Durch das Verlassen der conventionellen altchristlichen Tracht und Heranziehung der weiten sackartigen Wollgewänder seiner Zeit hat Giotto einen bedentsamen Schritt der Annäherung an die Natur vollzogen. Das eben besprochene Bild witderholt sich mit geringen Abweichangen auf f. 200, wo namentlich die Schlitzaugen der giottesken Profile auffallen.

Mit f. 207 beginnen die Commemorationen verschiedener Heiliger : eine Seite enthält die Antiphona und Oratio, die correspondirende Seite dagegen eine auf den jeweiligen Heiligen bezügliche bildliche Darstellung. In diese Darstellungen theilten sich die Meister, die giottesken Bilder scheinen aber auch nicht sämmtlich von einer Hand herzurühren.

F. 207', die Dreifaltigkeit, senesisch. Gott Vater auf einfachem Throne sitzend, hält im Schosse den an einem T-Kreuze befestigten Erlöser. Die Taube scheint auf den ersten Blick zu fehlen; sie findet 
sich bei schärferer Betrachtung am Querholze des Stammes flatternd, in dem sie sich in äusserst kleinen Verbälinissen kaum wahrnembar rom blauen Nimbus Gott Vaters abhebt. Dass aber der h. Geist in der That gemeint ist, beweist der Strahlennimbus um den Kopf des Vogels. Das Bild ist ziemlich abgerieben, wodurch die grüne Untermalung des Gesichtes Gott Vaters und des Leibes Christi nackt zu Tage tritt.

Das nächste Bild (f. 208) ist dem h. Geiste gewidmet, wie er auf Maria und die Apostel herabschwebt. Es ist ron einem giottesken Meister und hat auch etwas unter der Abreibung gelitten. Von gleicher Hand ist das folgende Bild (f. 209), die Auffindung des hl. Kreuzes darstellend. Die Kaiserin Helena tritt mit grossem Gefolge an eine Bergeshöhle heran, aus welcher zwei Knechte bemüht sind, das Kreuz herauszugraben. Ein weissbärtiger Maun, der die Arbeit zu leiten scheint, mag jener Judas sein, der nach der Legende durch Hunger gezwungen ward, den Bergungsort zu verrathen. Abweichend von der Legende erblicken wir in der Höhlung nur ein Kreuz. Auf dem Berge stehen grüne Bäume, worin mehr als eine blosse Andeutung der Landschaft liegt, aber der Hintergrund der Landschaft selbst ist in Rauten gemustert.

Auf f. 210 hat der Senese den h. Georg, den Drachen tödtend, dargestellt, auf f. 211 derselbe die Enthauptung des Täufers. Johannes beugt den Oberleib aus der Fensteröffnung eines Thurmes; unter dem Schwertstreich eines jungen Kriegers rollt der Kopf zu Boden. Einen woltuenden Gegensatz zu dem grün-fahlen Antlitz des Enthaupteten bildet die jugendlich-schlanke rosige Gestalt der Tochter der Herodias. welche zur Linken das Becken in Bereitschaft hält. Der Thurm is! rund, steigt in drei verjüngten Etagen anf und ist mit kouischer Kuppel überwölbt, vielleicht eine Reminiscenz ans Battistero des Diotisalvi.

Das nächste Bild - Petri Kreuzigung - ist wieder giottesk. Hier begegnen wir jenen rothen Spitzhärten, die der Meister so gerne seinen jugendlichen Gestalten gibt, dagegen erinnert ein hochragender langbärtiger Mann, der über das Hinterhaupt eine Kapuze geworfen hat - vermuthlich der prefectus Agrippa der Legende - lebhaft an die tiefernsten Gestalten der Capella degli Spagnuoli. Der Heilige wird, die Füsse zu oberst, mit Stricken am Kreuze festgebunden, der jugendliche Kaiser sieht zur Linken der Handlung zu.

F. 213 bringt die Marter des h. Laurentius von der Hand des Senesen. Der Heilige liegt nackt ausgestreckt auf dem Feuerroste; Kaiser Decius sieht rom Throne zu und macht befehlende Geberden 
gegen die Schergen, deren einer den Heiligen mit der Feuerzange niederpresst, während der andere mit einem Blasebalg das Feuer anfacht. Zur Rechten des Kaisers steht der Präfect Valerianus der Legende; oben aus einem Rund der Architectur segnet der Erlöser herab.

Von gleicher Hand ist eine Darstellung aus dem Leben des h. Nicolaus (f. 214). Unter einem gothischen Tabernakel liegt ein Mann im Bette; der aufrecht stehende nackte Oberkörper ist sichtbar. Von drei Frauen wird er betreut: eine lässt ihn aus einem Fläschchen trinken, die zweite faltet die Hände und blickt mitleidig auf den Kranken, die dritte aber ist dem Heiligen zugewendet, welcher als jugendlicher Mönch (mit Tonsur, aber ohne mönchische Kleidung) von rechts herankommt; in der Rechten hält er ein Buch, in der Linken eine goldene Traube. Die Darstellung bezieht sich wol auf die Rettung der drei Töchter des dürftigen Nachbars von der Schande. Dass der Heilige, der doch Bischof von Myra war, ohne die Abzeichen seiner Würde dargestellt wurde, erklärt Petrus de Natalibus aus einer thätlichen Ausschreitung, die sich der Heilige auf dem Nicaener Concil gegen einen Arianer hatte zu Schulden kommen lassen und die ihn um Mitra und Pallium brachte ${ }^{1}$ ).

Auf f. 215 hat der Senese den Märtyrertod des h. Dionysius mit seinen beiden Gefährten Rusticus und Elentherius dargestellt. Nach der Legende werden sie bei einer Mercurstatue mit Beilen erschlagen ${ }^{2}$ ). Im Bilde wird nur der Heilige selbst mit einem Beile getödtet, die Gefährten mit Schwertern. Auch die Bildsäule ist nicht sichtbar.

Gleichfalls von Senesen ist auf f. 216 der Tod des h. Thomas von Canterbury dargestellt. Der Heilige kniet betend, die Mitra liegt am Boden. Der Mörder sind der Legende ${ }^{3}$ ) entsprechend vier, und zwar gepanzert (loricati).

Auf f. 217 empfängt der h. Franciscus von Assisi die Wundmale Christi; neben ihm sitzt ein lesender Mönch, den landschaftlichen Hintergrund geben eine Bergeshöhle nit Bäumen und ein 'Häuschen. Das Bild ist giottesk, verräth aber in den Gesichtern eine etwas verschiedene Malweise, was auf eine zweite, wenngleich im Uebrigen völlig verwandte Hand schliessen lassen könnte.

1) I, 28 (venezianische Ausgabe von 1506): Fertur Nicolaum iam senem Niceno concilio interfuisse et quendam Arrianum zelo fidei in maxillam percussisse obidque a concilio mitra et pallio privatum extitisse, propter quod at plurimum sine mitradepingitur. 2) Jacobus a Voragine: iuxta jdolom Mercurii cum securibus trium capita preciduntur.. э) Surius, Vitae sanctorum (ed. Colon. 1581) 1164. 
In der Behandlung ganz nahe steht das folgende Bild (f. 218): de reliquiis ystoria. Unter einem reichen gothischen Tabernakel stehen auf einer mensa ein Vortragkreuz und ein rundförmiges Reliquiar. Ein weiteres Vortragskreuz bält rechts ein König, von welchem später die Rede sein soll, links singen Mönche aus Notenbüchern.

F. 219 ist vom Senesen gemalt. König Ludwig der Heilige bietet knieend den Trinkbecher einem Mönche, der an einer mit Speisen und Krug besetzten Tafel sitzt. Hinter dem König steht ein anderer Mönch. In einer der Lebensbeschreibungen des Königs wird uns erzählt, dass er Arme und Mönche speiste und selbst bediente. Da auf dem Tische eine Brodscheibe und ein Fisch auf einer Schüssel deutlich erkennbar sind, wird man an eine ebendort äberlieferte Nachricht erinnert, dass der König den Mönchen eines Cisterzienserklosters am Freitage Brod, Fische und Wein spendete ${ }^{1}$ ).

F. 220 ist senesisch und stellt eine Episode aus dem Martyrium der h. Katharina von Alexandrien dar. In der Mitte des Bildes kniet die Heilige, den Blick nach oben gewendet, mit gefalteten Händen. Der Himmel ist in blauen und rothen, ron Licht zu Schatten abgetonten Wolken dargestellt, die palmettenartig in einander greifen. Aus ihnen regnet Feuer und ragt die Hand Gottes herror, das Doppelrad zerbricht und am Boden liegen die Erschlagenen. Die Legende spricht von vier Rädern und lässt einen Engel das Zerstörungswerk vollenden. Im Ganzen aber schliesst sich das Bild an die Legende an, die bei Jacobus a Voragine und bei Petrus de Natalibus²) völlig gleich lautet. Es ist dies eine der besten Compositionen, auf Goldgrund meisterhaft ausgeführt. In den Erschlagenen begegnen uns bemerkenswerthe Versuche in Verkürzungen. Inmitten des Grauses blickt die schöne Jungfrau gottergeben zum Himmel ${ }^{3}$ ).

Auf f. 221 soll folgen: de sancta Margarita ystoria, im Gebete aber wird Maria Magdalena angerufen, und diese hat der Senese auch im Bilde dargestellt. Sie kniet, eine liebreizende Erscheinung, in der

1) Acta Sanct. der Boll. August 5, 595: Saepe etiam diebus veneris manducabat in triclinio ad mensam abbatis ... et semper, quando illic manducabat, ad victum dabat conventui panem et $v$ in $\mathrm{m}$ ac bis gemina fercula piscium. 2) $\mathbf{X}, 105$. Prefectus quidam ei (regi) suasit, ut infra triduum quattuor rotas serris ferreis et gladiis circumseptas prepararet, ut tam terribile tormentum puelle membra dissecaret... quod et factum est. Tunc virgo beata dominum exoravit, ut ad conversionem circumstantis populi ipsam machinam dissiparet. Et ecce angelus domini molem ipsam tanto impetu divellendo concussit, quod quattuor milia gentilium interemit. $\left.{ }^{3}\right)$ An die Darstellungen der h. Katharina und Magdalena hat Simone Martini besondere Sorgfalt gewendet. vgl. Crowe und Cav. ", $238,241$. 
Landschaft, die durch Bäume und Gräser individualisirt ist, vor ihr erscheint zur Rechten der auferstandene Christus.

Von selber Hand folgt auf f. 222 die Illustration zu: de omnibus sanctis ystoria. Ven rechts und links treten Heilige heran, von denen nur die vier vordersten völlig sichtbar sind; unter ihnen ist nur Paulus dentlich attribuirt. Die übrigen drei sind ein Mönch, ein Bischof und ein Weib; sie sollen wol nur Generalisirungen sein, ohne dass der Künstler bestimmte Persönlichkeiten im Auge hatte.

Auf f. $223^{\prime}$ folgt das Bild des h. Ludwig von Toulouse, eines der trefflichsten von der Hand des Senesen (Abb. 1). Von ihm wird weiter unten die Rede sein.

Auf f. 224' hat ein Giottesker die Marter des Evangelisten Johannes dargestellt (Abb. 2 S. 442). Der Heilige steht mit gefalteten Händen nackt im Oelkessel, ein Scherge schürt das Feuer, ein anderer facht es mit dem Blasebalg an, ein dritter giesst dem Heiligen aus einem Kruge siedendes Oel über den Kopf. Letztere Bewegung in Zusammenhalt mit der Stellung des Johannes lässt eine Darstellung der Taufe Christi als Vorbild vermuthen. Links sitzt Domitian auf dem Throne, ihm zur Linken einer seiner Vertrauten. Schulz bespricht ein giotteskes Gemälde in der Capella Brancaccia in S. Donenico in Neapel folgendermassen 1 ):,$\ldots$ befiehlt ein auf seinem Throne sitzender von Schergen umgebener Tyrann (Domitian) die Marter des Heiligen im siedenden Kessel. Johannes halb sichtbarer nackter Körper ist nicht besonders gezeichnet."

F. 225 hat ein Bjld von senesischer Hand, das die Geschichte des h. Nicasius illustriren soll. Der Heilige steht an der mit einem Kelch besetzten Mensa und hält seinen bluttriefenden Kopf in der Hand; ein Engel umfasst ihn schirmend. Nach der Legende wurde dieser Bischof von Rouen von den Vandalen enthauptet; weder Surius noch Jacobus a Voragine und Petrus de Natalibus wissen etwas zu erzählen, worauf sich unsere Darstellung bezöge. Dagegen wird vom h. Dionysius erzählt, dass er unmittelbar nach seiner Enthauptung sich erhoben und unter Führung eines Engels sein eigenes Haupt vom Montmartre nach dem späteren St. Denis getragen habe?2). Möglicherweise liegt hier irgend eine Verwechslung vor, deren sich der senesische Künstler umso leichter schuldig machen konnte, als ihm

1) H. W. Schulz, Denkmäler der Kunst des Mittelalters in Unteritalien s, 168. 2) Jac. a. Voragine: Statimque corpus s. Dionysii se erexit et caput suum inter brachia angelo duce et celesti lumine precedente per duo miliaria deportarit. 
die Vertrautheit mit den Legenden franzöșischer Localneiliger naturgemäss fehlen musstẹ,

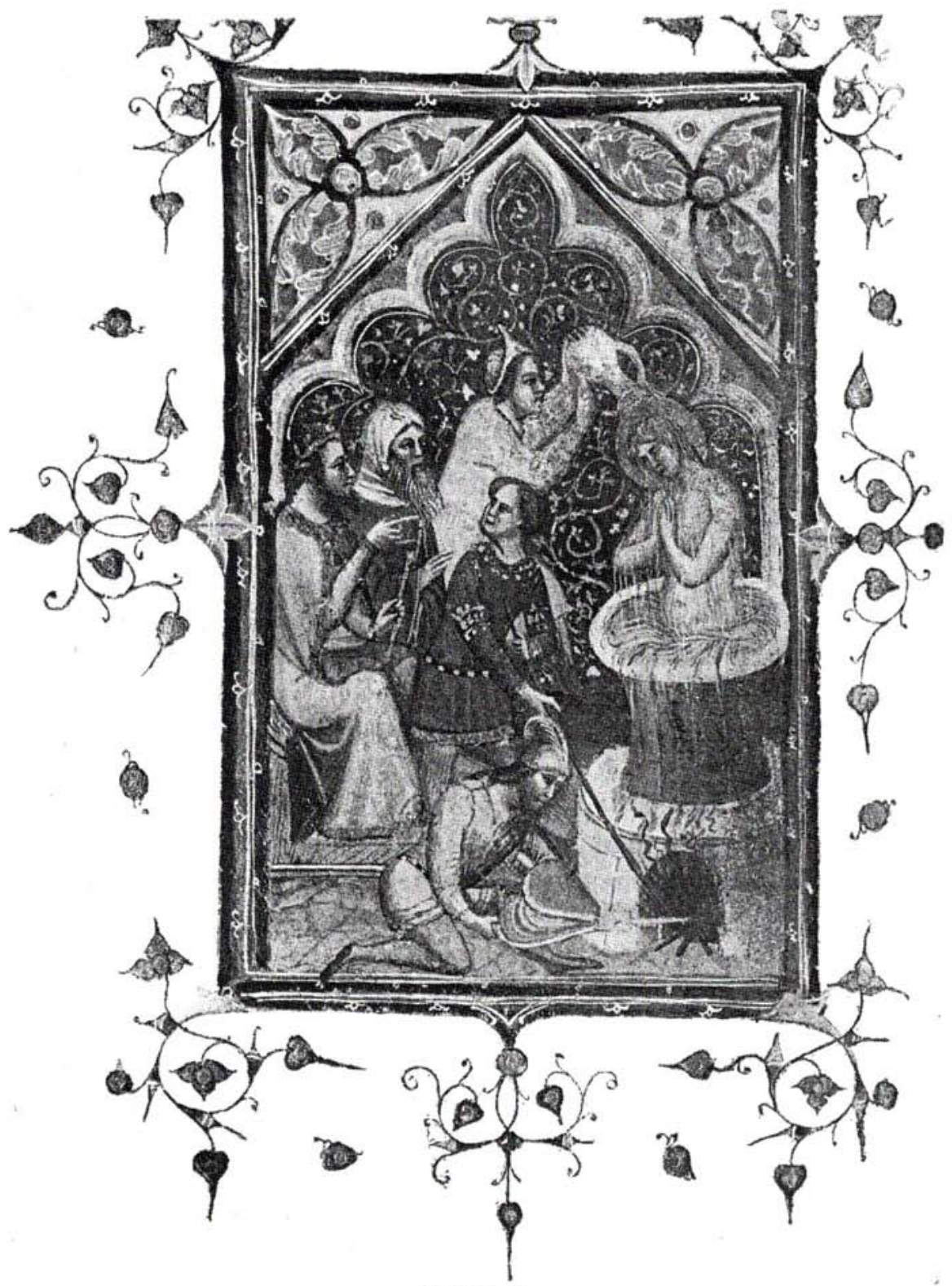

Abbildung 2.

Die Heiligen-Commemorationen schliessen mit einer Darstellung aus dem Leben der h. Elisabeth, die der Senese auf f. $226^{\prime}$ wibietet. Die Heilige liegt schlafend im Bette, mit der Rechten presst sie Rosen 
an die entblösste Brust. Von links naht ein junger Mann - ihr Gremal -, der mit der Rechten die Decke berührt. Es ist wol die Wandlung der für die Armen bestimmten Brote in Rosen gemeint, als der Gemal, der ihr diese Schenkungen verboten hatte, sie überrascht. Auf einer Bank liegt ein Kanm und ein Paar rother Pantoffeln; ebenso genrehaft natürlich ist das Bettzeug dargestellt. Nach hinten ist die Scene durch einen Vorbang abgeschlossen; das Antlitz der Heiligen ist von ausserordentlicher Schönheit.

Von f. 228--247 reichen die Gebete für die canonischen Stunden. Die beigegebenen Bilder sind sämmtlich giottesk. Das erste (f. 228) führt uns die Geburt Christi vor: die Wöchnerin liegt im Bette und streckt die Arme nach dem Kinde aus, das in der Wiege (d. i. in der Krippe) zu ihrer Rechten rubt. Esel und Ochs lagern daneben auf dem Boden. Nordwärts der Alpen pflegte man die Wöchnerin durch doppelten - quadratischen und runden - Nimbus auszuzeichnen, die Krippe brachte man über dem Fussende des Bettes an, darüber die Thiere. Die Anordnung ist hier somit eine abweichende. Rechts sitzt Joseph, das Kinn auf die Rechte gestützt. Schauplatz ist nicht ein gezimmerter Stall, sondern eine Höhle, durch deren Oeffnung der Stern hereinleuchtet.

Das Gebet zur Terz zeigt wieder die früher erwähnte Königin mit den Dienerinnen; ron links schwebt die Dreieinigkeit heran: Gott Vater bält in den Armen vor sich das Jesuskind, auf dessen Haupt vom Munde des Vaters die Taube niederflattert. Das Gefolge bilden schwebende Engel mit verschränkten Armen.

Mit geringen Abweichungen bietet dasselbe das Bild zur Sext (f. 234'). Aus dem Munde des Kindes springt ein Gnadenstrahl auf die Königin herab, die sich in anmuthiger Bewegung vorbeugt. Zur Non (f. 237) wird die h. Messe dargestellt. In rundbogiger Pfeilerhalle steht ein Mönch celebrirend am Altar, über den Rücken des langen rothen Messkleides läuft ein goldenes Kreuz. Ihm assistiren zwei Mönche in weissen und blauen Gewändern, an denen die rundlich modellirten Parallelfalten - charakteristisch für die italienische Malerei bis auf Fra Angelico hinab - besonders augenfällig hervortreten. Die rechte Hälfte des Bildes ist eingenommen von der Königin und ihren Dienerinnen: sie knieen unter einem Baldachin aus blauen liliendurchwirkten Vorhängen. Von der Decke streuen zwei Engel Rosen auf die Scene.

Auf f. $240^{\circ}$ folgen die Gebete respere de beata virgine Maria. Diesmal sehen wir die Madonna zur Rechten sitzend, die Rechte segnend erboben, in der linken ein Buch; links kniet die Königin mit 
den Dienerinnen; sie wird ron einer Heiligen als Fürbitterin der Madonna empfohlen. Der gänzliche Mangel an Attributen lässt die Persönlichkeit der Heiligen nicht erkennen.

Die Reibe dieser Gebete schliesst auf f. 244' mit dem Completorium ab. Dargestellt ist die Krönung Mariae, wobei aber Christus seine neben ihm sitzende Mutter nur segnet, während die Kröuung vou einem senkrecht herabsehwebenden Engel vollzogen wird. Rechts und links sind musicirende Engel.

Das folgende Officium passionis domini nostri Jesu Christi ist mit zwei giottesken Bildern ausgestattet. Auf f. 248 wurde die Gefangennehmung Christi dargestellt: Kriegsknechte ergreifen den Herrn, die Nacht ist durch goldene Sterne auf blauem Grunde und durch brennende Fackeln angedeutet.

F. 254 zeigt in figurenreicher Composition die Verspottung Christi.

Zum Officium de spiritu sancto ist auf f. 273 die Taufe Christi vom Senesen noch ganz in der unperspectivischen älteren Weise dargestellt. Christus steht röllig entkleidet im Wasser, das ihm bis an die Hüfte reicht. Links kniet der Täufer, rechts zwei Engel mit Tüchern. Die Taube schwebt berab vom Himmelsgewölbe, das durch einen schmalen blauen Streifen mit goldenen Sternen angedeutet ist. Ein Baum bezeichnet die Landschaft; der Hintergrund ist in Rauten mit Lilien gemustert.

Auf f. $293^{\prime}$ beginnen die sieben psalmi poenitentiales mit dem giottesken Bilde der Auferstehung auf Goldgrund. Die Composition ist durch einen blauen Bogen zweigetheilt: oberhalb derselben thront in der Mitte der Weltenrichter, ihm zunächst steheu rechts and links je ein Engel mit den Marterwerkzeugen, in zweiter Linie links Maria, rechts Johannes. Unterhalb des Bogens erheben sich die Todten aus den Särgen, während zwei Engel die Posaunen erschallan lassen. Die Durchführung ist schwächer.

Auf f. 301 beginnt das Officium defunctorum mit einer Todtenmesse an der Spitze von giottesker Hand. Hinter einem mit rothen weiss- und goldgemusterten Tuche bedeckten Katafalk liest ein Mönch die Todtengebete aus einem Buche, das er in der Linken hält, wăh rend die Rechte den Weihwedel schwingt. Ihm assistirt ein junger Mönch mit Kreuz und Weihbrunnkessel, links beten zwei vermummte Frauen.

Es wurde bereits eingangs erwähnt, dass die letzten Folien des Codex 328-347 in Schrift und Miniaturen abweichen und vielmehr 
französischen Charakter verrathen. F. 328 zeigt eine völlige Umrahmung in der Weise, wie sie in Frankreich in der zweiten Hälfte des 14. Jahrh. aufkam: gothisches Laubgeschlinge, das sich gelegentlich zu einem Oval öffnet, für Aufnahme bildlicher Darstellungen so im vorliegenden Falie für eine Anbetung des Kindes durch die Madonna und für eine Verkündigung. Auch die Initialen, die im italienischen Theile der Handschrift durchaus des figürlichen Schmuckes entbehrten, werden hier mit solchem ausgestattet, so auf f. 328 mit den drei göttlichen Personen und der Madonna, wobei der Hintergrund Lilien und Rauten zeigt. Die reichliche Anwendung von schwarzen Umrissstrichen spricht für einen Franzosen, dem noch die ältere Weise geläufig war. Auch die ornamentale Ausfüllung der Initialen ist nordisch. Auf f. $342^{\prime}$ ist ein Bild von St. Anna selbdritt auf gothischem Thronhimmel; danit ist der figürliche Schmuck dieser Schlussgebete erschöpft.

Erscheint nach dem Gesagten unzweifelhaft, dass der Codex zum allergrössten Theile von italienischen Händen geschrieben und illuminirt wurde, so müssen wir uns fragen, was Denis auf die Vermuthung französischen Ursprungs hatte bringen können? Es ist auch zuzugeben, dass die inneren Merkmale, nach denen man die Provenienz solcher Handschriften zu bestimmen pflegt und auf die man sich zu Denis' Zeiten im Allgemeinen besser verstand, als auf die äusseren, vorwiegend auf Frankreich hinweisen. Es sind dies vor Allem die Heiligennamen des Kalenders, unter welchen Denis zahlreiche französische Localheilige fand, ohne dass ihm allerdings entging, dass eine spätere Hand eine Anzahl nordischer Heiligennamen hinzugefügt hat. Da Denis ferner bemerkte, dass auf dem angeblich fünf Mal wiederholten Bilde einer adorirenden Königin Sammtkissen und Vorhang mit gelben Lilien auf blauem Grunde geschmückt seien, zog er daraus den Schluss, der Codex müsste ursprünglich für ein weibliches Mitglied des französischen Königshauses geschrieben sein., Die nordischen Zusatznamen liessen Denis unzweifelhaft einen späteren schwedischen Besitzer annehmen und, da der Codex erst im 18. Jahrh. von Hall in Tirol nach Wien gekounmen war, ergab sich die fernere Hypothese, das Buch dürfte im Gefolge der Königin Christine nach Tirol gekommen sein (1655). Wir wollen zunächst von allen späteren Zusätzen absehen und versuchen auf Grund der inneren Merkmale, vorerst der bereiits von Denis verwertheten, dann der ihm entgangnen die Provenienz festzustellen.

Auch hier möge der Kalender mit den Heiligennamen den Anfang machen. Ausser den altchristlichen Heiligennamen, wie sie. 
durch die Martyrologien und Heiligenverzeichnisse berkōmmlich überliefert Gegenstand gemeinsamer Verehrung der ganzen Christenheit geworden sind, finden wir zahlreiche Localheilige, die bis auf eine einzige Ausnahme nicht Italien, sondern Frankreich angehören. Blos die Erwähnung des Gedächtnisstages der Translatio 8. Nicolai (nach Bari 1087) weist auf die apenninische Halbinsel; die übrigen Heiligennamen vertheilen sich anf sämmtliche Erzdiöcesen Frankreichs, doch weitaus am meisten erscheint der Sprengel von Sens begünstigt, wovon wieder die Hälfte auf Paris entfällt. Aber auch die nördlich angrenzenden lothringischen und niederländischen Landschaften (Metz, Lüttich usw.) sind vertreten, dagegen äusserst spärlich die Provence und die Gascogne. Der Schwerpunkt erscheint somit in den Norden verlegt, dessen Mittelpunkt die Isle de France, der Hauptsitz des französischen Königshauses und die Wiege der Gothik bildet. Entscheidend ist hiefür auch der Umstand, dass zum 25. August mit rothen Buchstaben das Fest König Ludwigs des Heiligen verzeichnet ist, womit wir zugleich den ersten Ansatz für die Zeitbestimmung gewinnen, indem die Canonisation Ludwig IX. in's Pontificat Bonifaz VIII. (1296) fällt.

Werden wir durch die Untersuchung der Heiligennamen zu demselben Resultate wie Denis geführt, so wird uns auch jene weibliche Gestalt mit der Lilienkrone, knieend auf liliengeschmücktem blauen Kissen vor ebenso geschmücktem Vorhange, mit Denis den ursprünglichen Besteller des Codex in dem Kreise des französischen Königshauses oder wenigstens eines mit demselben verwandtschaftlich zusammenhängenden Königshauses suchen lassen. Die Lilien wiederholen sich auch auf den in Rauten gemusterten Hintergründen mehrfacher bildlicher Darstellungen.

Eine bestimmte Lösung bietet uns aber das Bild des h. Ludwig von Toulouse aut f. 223 mit der Beischrift: Istoria beati Ludovici, clare memorie domini regis Roberti fratris. Dieser Sohn König Karl II. von Anjon und Bruder König Roberta des Weisen von Neapel war in jungen Jahren in den Franciscanerorden eingetreten, wurde dem Bisthum von Toulouse vorgesetzt, starb aber bereits 1298 im Alter von kaum 24 Jahren $^{1}$ ). Auf unserem Bilde (Abb. 1) ist er dargestellt

7) Seine Lebensbeschreibung ist im 2. Band des August der Acta Sanctorum (p. 775-822) abgèdruckt. Eine neuere Arbeit über den Heiligen vom Padre Ludovico da Palma, Napoli 1855, blieb mir unzugänglich. Mit Benuteung des Materials aus den angiovinischen Registern hat in vortrefflicher Weise Camillo 
in der Franciscanerkutte, über welche er den blauen liliengeschmückten bischöflichen Mantel mit edelsteinbesetztem Goldsaum geworfen hat, das jugendliche, ja knabenhafte Antlitz bedeckt mit der Mitra, verklärt vom Nimbus. In der Rechten hält er den Krummstab, in der Linken die Krone, auf welche er durch das Ordensgelübde verzichtete. Die äusserst fein und sorgfältig ausgeführte Figur steht unter einem Tackeubogen 1), von einem krabbenbesetzten Windberg uberstiegen und flankirt von fialenbekrönten Strebepfeilern, eine bunte und prächtige A rchitectur, die in einen Dornblattrahmen hineincomponirt ist ${ }^{2}$ ). Die Canonisation dieses Prinzen erfolgte am 7. April 1317 unter Johann XXII. Da sich der Name des Heiligen zwar im Kalender zum 19. August findet, aber nicht von der Hand des ursprünglichen Schreibers, sondern von jener des nordischen Ergänzers, möchte man annehmen, dass der Kalender noch vor 1317 geschrieben worden sei. Erscheint dies bei dem Umstand, als Schreiber und Illuminatoren dem ganzen Codex gemeinsam sind, unwahrscheinlich, so müssen wir auf eine andere Lösung bedacht sein, die sich in der That darin bietet, dass man wol dem italienischen Schreiber ein französisches Gebetbuch sammt Kalender zum Copiren übergab: der Italiener hatte nur abzuschreiben, was sich in seiner Vorlage vorfand; diese musste allerdings nach 1296, der Canonisirung Ludwig IX., aber vor 1317, derjenigen des tolosanischen Heiligen geschrieben sein. Auch die den Psalter mit den Canticis abschliessende Litanei (f. 181') kennt nur einen b. Ludwig. Dass aber diese Litanei, die noch zum Psalter gehört, gleichzeitig mit den folgenden Gebeten, worunter die Commemorationen der Heiligen, also auch diejenige des $h$. Ludwig von Toulouse geschrieben wurde, beweist unmittelbar das Quaternionengefüge. Der Schreiber schrieb nach Quaternionen und Quinternionen ${ }^{3}$ ),

Minieri Riccio im Archivio storico per le province napoletane, Band VII Genealogia di Carlo II d'Angiò p. 58-67 über den Prinzen gehandelt.

1) Eine namentlich bei Simone Martini beliebte Architectur. ' Ausser den noch vorhandenen Darstellungen des Heiligen in S. Lorenzo und im Refectorium von Santa Chiara werden von Summonte (Historia di Napoli 1601, 2, 345) als zu seiner Zeit noch erhalten in der angiovinischen Hauscapelle im Dom zu Nespel erwăhnt: Dipinti sulle mura i fatti della vita ed i miracoli operati dal santo. Aus den Registern führt Riccio (a. a. 0. 64) ein, reliquiario lavorato ad jmaginem b. Ludovici de Massilia cum mitria Croczis lapidibus pretiosis et perlis` an. 3) Schulz a. a. 0. 4, 106 Reg. $n^{0} 278$ erwähnt einen Schreiber Joh. de Niellis, der nach Quaternionen bezahlt wird. Das Papier kaufte man ebenfalls nach Quaternionen (Arch. napol. 8, 19.7 Regest zum 26. Jan. 1337), wobei man quaderni grandi und piccoli unterschied (ebends p. 383 Regest zon 14. Ang. 1841), was wol aufs Format zu denten ist. 
nur der Kalender bildet einen Sexternio für sich, da jeder Monat ein Blatt umfasst. Die Hefte sind zwar nicht nummerirt, aber durch die Custoden bezeichnet, so dass sich bei gleichzeitiger Untersuchung des gegenwärtigen Verbandes die ursprüngliche Anlage genau controlliren lässt. Danach fiel das Ende des Psalters und der Anfang des Liber precum in einen und denselben Quinternio.

Die erwähnte Beischrift zum Bilde des h. Ludwig gibt uns noch weitere Aufschlüsse. Zunächst frägt es sich, auf wen sich der Beisatz "clare memorie "beziehe. Bezieht er sich auf Robert, daun wäre die Handschrift erst nach dessen Tode (1343) verfasst. Der Beisatz kann sich aber auch auf fratris, somit auf Ludwig beziehen: für beides sind Gründe beizubringen. Für die erstere Annahme scheint die Stellung im Satze zu sprechen. Nach Ludovici folgt ein Komma, und wir würden clare memorie für Ludwig eher nach istoria suchen. Dagegen spricht jedoch, dass auf f. 218 - der oben erwähnten Illustration zu: de reliquis ystoria - ein König dargestellt ist mit der Lilienkrone auf dem Haupte, in langem dunkelblanen Talar : eine Gestalt, auf die völlig die Beschreibung passt, die Schulz (a. a. 0 . 3, 156) vom Bilde des Königs Robert in San Lorenzo zu Neapel von der Hand des Simone Martini entwirft: ,.... als solchen bezeichnet ihn das scharfe individuell ausgeprägte Gesicht, die grosse gebogene Nase, die gefurchten Wangen, die niedere Stirn, das spitze vorspringende Kinn, der magere lange Hals. Sein Gewand ist eine dunkelblaue Stola mit breitem rothen Bande eingefasst und mit goldenen Lilien durchwirkt." Die Beschreibung stimmt bis aufs Gewand, blos der rothe Saum und die Lilien sind weggelassen, sie fehlen aber in unserem Codex ebenfalls dem blauen Talar König Ludwigs des Heiligen (f. 219). Vergegenwärtigt man sich, dass nach Roberts Tod kein König, sondern eine Königin - Johanna I. - bis 1381 den Thron der neapolitanischen Angiovinen inne hatte, so wird man umso geneigter sein, das in Rede stehende Bild auf König Robert zu beziehen. Was aber jenes öfters wiederkehrende Bild einer Königin in unserem Codex betrifft, müssen wir in der'Deutung zwischen Sancia, der zweiten Gemahlin Roberts, die im Jahre 1345 gestorben ist, und zwischen Johanna I. schwanken. Für letztere sprechen, wie wir noch sehen werden, einige Verwandtschaften mit einer bildlichen Darstellung ihrer Vermählung mit Ludwig von Tarent in der Incoronata. Wie dem auch immer sei, schon aus stilistischen Gründen wird es uns unmöglich, weit über das Todesjahr Roberts des Weisen (1343) herabzugehen. Die Abfassung muss etwa zwischen 1330 und 1350 liegen und zwar muss sie auf Veranlassung des Hofes von Neapel geschehen sein. Man könnte 
allerdings einwenden, dass auch das französische Königshaus italienische Illuminatoren beschäftigte ${ }^{1}$ ); doch bricht in diesen Fällen stets mehrfach das Französische hindurch, zumindest in der Schrift. Andrerseits liegen gerade für die Zeit Roberts des Weisen ganz genaue Nachrichteu über die Thätigkeit florentinischer und senesischer Meister in Neapel vor.

Die Geschichte der Malerei in Neapel befand sich bis in die neueste Zeit infolge der Fälschungen B. de Dominici's in arger Verwirrung. Dieser hatte die älteren Kunstwerke Neapels auf selbsterfundene einheimische Künstler zurückgeführt und durch mehr oder minder geschickte Quellenfälschungen das allgemeine Urtheil trotz augenfälligen Zusammenhanges der angiovinischen Malereien mit den Denkmälern der toskanischen Malerei in bestimmtester Weise 7.0 beeinflussen gewusst. Erst die historisch-kritische Betrachtungsweise unseres Jahrhunderts brachte bedenkliche $Z$ weifel gegen die Zuverlässigkeit de Dominici's an den Tag und es ist in dieser Beziehung neben Catalani und Hettner namentlich Schulz vieles Verdienst beizumessen. Als vollends Minieri-Riccio, der vor kurzem verstorbene Director des neapolitanischen Staatsarchivs, seine Regesten aus den angiovinischen Registern veröffentlichte, gewann man die Belege für ein ganz anderes Bild des neapolitanischen Trecento, welches von Faraglia $\left.{ }^{2}\right)$ eine übersichtliche Darstellung gefunden hat. Es ist hier nicht der Ort, um auf diese Ergebnisse im einzelnen einzugehen; im allgemeinen lässt sich sagen, dass neben einer untergeordneten, mehr lernenden Antheilnahme der Einheimischen unter Robert dem Weisen die Vertreter der toskanischen Malerei die Führung in der Kunst Neapels innehatten. Giotto selbst mag nur im Castel Nuovo gemalt haben; zu den schon von Schulz veröffentlichten urkundlichen Belegen der Wirksamkeit dieses Florentiner Hauptmeisters in Diensten der Angiovinen gesellt sich ein neuer ${ }^{3}$ ), dem wir nicht nur eine genaue Aufzählung der von Giotto gebrauchten Malutensilien entnehmen, sondern auch die Kunde, dass unter ihm eine Anzahl diversorum magistrorum tam pictorum quam manualium et manipulorum gearbeitet haben muss. Wenigstens einige der ersteren mögen mit Giotto aus

1) Woltmann, Gesch. der Malerei 1, 476 führt mehrere solche Falle an. Ebenda p. 474 finden sich auch einige für die neapolitanischen Angiowinen gefertigte Bilderhandschriften aufgezählt. Beiderseits finden sich Hinweise auf. Ver-. wendung senesischer Künstler. 2) Im 7. und 8. Bande des Archivio storico napoletano unter dem Titel: Le Memorie degli artisti napolitani pübbljcate da B. de Dominici. 3) Arch. nap. $7,676 \mathrm{znm} 20$. Mai 1331. 
Florenz gekommen sein. Von diesen Malereien im Castel Nuovo ist bekanntlich nichts erhalten, wol aber Werke von Giotto's Schulern, namentlich im Refectorium ron Santa Chiara und in der Incoronata. Das Fresco in Santa Chiara ${ }^{1}$ ) zeigt nach Abbildung und Beschreibung keinerlei augenfällige Anslogien mit den Bildern unseres Codex, mehr dagegen das auf Tafel 76 bei Schulz publicirte Fresco der Incoronata, die Vermählung der Königin Johanna I. mit Ludwig von Tarent im Jahre 1351. Die langen Hängeärmel und die völlige Büste der Johanna sind auch der knieenden Königin unseres Codex eigenthümlich. Weniger mag die gemeinsame Lilienkroue besagen, die ja bekanntlich von der Kunst ebenso zum Attribute aller Könige gemacht worden ist, wie die Bienenkörbe der Chigi zum Merkzeichen päpstlicher Stiftungen. Wichtiger ist die Uebereinstimmung in der Kopftracht der Begleiterinnen; auch die rothen Spitzbärte der Männer weisen auf eine gemeinsame Modesitte der Zeit. Der auf das Brautpaar senkrecht herabschwebende Engel ist in unserem Codex ebenso verwendet für die Krönung Mariae. Noch 1371 bat Nicola di Tomaso aus Florenz in S. Antonio abbate das Bild dieses Titelheiligen gemalt.

Steht die Wirksamkeit Giotto's in Neapel urkundlich fest, so kann man dasselbe nicht mit gleicher Sicherheit von dem Hauptmeister der zweiten toskanischen Schule, der senesiscben, behaupteu. In San Lorenzo wird das Bild der Krönung des Königs Robert durch den h. Ludwig von Toulouse gezeigt, welches durch eine Inschrift als Werk des Simone Martini bezeichnet ist. Nach dem stilistischen Charakter des Bildes haben die Kunsthistoriker ziemlich einstimmig die Echtheit dieses Tafelbildes auerkannt und auch Faraglia hat sie bedingungslos angenommen. Nur Minieri Riccio ${ }^{2}$ ) scheint die Echtheit der Inschrift zu bezweifeln, da er das Bild einem mastro Simon cremonese zuschreibt, worin er sich dem C. d' Engenio (Napoli sacra p. 115) anzuschliessen scheint. Doch ist das Zeugniss des kritiklosen d'Engenio, der noch den fabelhaften Masuccio für baare Münze nimmt, zu schwach, um dem Urtheil Riccio's den Vorzug vor denjenigen Faraglia's und der deutschen Forscher einzuräumen. In techniseher Beziehnng ist das Bild unzweifelhaft als senesisches Werk schon von Schulz ${ }^{3}$ ) nachgewiesen. Die Betrachtung dieses Bildes, von dem ich leider keine Publikation namhaft zu machen im Stande bin, ergibt nun unzweifelhaft, dass dasselbe unserem senesischen Miniaturisten

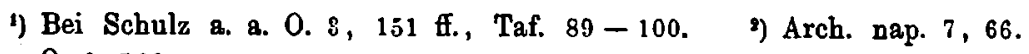
8) a. a. $0.3,166$. 
zum Vorbilde gedient haben muss: in beiden Fällen dieselbe dem Beschauer zugewandte Jünglingsgestalt, mit gleichen Gewändern und Attributen; nur ist er im Tafelbilde thronend dargestellt und unterhalb der Krone der knieende Robert eingefügt; durch letzteren erhält aber der Gestus mit der Krone, der in der Miniatur unverständlich bleibt, erst seine Erklärung. Durch Hinweglassung Roberts und zweier krönender Engel hat der Illuminator sein bescheideneres Bild zu vereiufachen gesucht.

Wenn die Fresken in der Martinscapelle in San Francesco zu Assisi wirklich von Simone's Hand stammen, so ist durch die daselbst befindlichen Angiovinenbilder ein weiterer Beweis für die Beziehungen des Meisters zu jener Dynastie gewonnen 1 ).

Lassen uns die Register für die Thätigkeit des Simone di Martino in Stiche, so liefern sie desto umfangreichere Nachrichten für die ausgebreitete Verwendung eines anderen senesischen Künstlers in König Roberts Diensten. Gino da Siena, dessen Thätigkeit in Neapel bereits von Schulz für die Jahre 1323 - 1329 nachgewiesen worden war, erscheint nach den Registern ${ }^{2}$ ) auch über das Jahr 1329 hinaus in angiovinischen Diensten in hervorragender Weise thätig. Nach einer Rechnung vom 2. Januar 1338 ${ }^{3}$ ) war der Meister an diesem Tage bereits todt, muss aber am 8. Juli 1336 noch am Leben gewesen sein. Durch diese Daten wird die durch Milanesi vertretene Identität des Meisters mit Tino di Camaino, der 1339 gestorben sein soll, sehr in Frage gestellt. Bei der Universalität der Künstler jener Zeit ist es an sich wahrscheinlich, dass Gino da Siena, der sonst überall Architect genannt wird, auch Maler gewesen ist. Wenigstens möchte darauf ein Regest4) schliessen lassen, worin der Meister zum direttore delle pitture einer offenbar von ihm selbst erbauten Capelle ernannt wird. Auch sonst ist die Verbindung der Angiovinen mit den Künstlerkreisen von Siena bezeugt. Am 2. Januar 1338 wird der Glockengiesser Giorgio da Siena nach Neapel berufen, um daselbst einige Glocken zu giessen ${ }^{6}$ ). Noch im Anfange des 15. Jahrh. ist Matteo da Siena in Neapel thätig.

Auch für das Schriftwesen und die Miniaturmalerei unter den Angiovinen hat Minieri-Riccio dankenswerthes Material beigebracht.

1) Crowe und Cav. 2, 242 ff. $\quad$ 2) Arch. nap. 8, 12, 15, 22 2u den Jahren 1884-1385. 3) Arch. nap. 8, 205. \) Arch. nap. 8, 12. Was es mit dem in einem Regest vom Jahre 1832 Arch. 7, 683 und rom 26. Jan. 1837 ebenda 8, 197 als gekauft erwähnten Apparatus magistri Cini super codice für eine Bewandtniss hat, gelang mir nicht festzustellen. 5) Arch. 8, 204. 
Aus zahlreichen Regesten ersehen wir ein fürsorgliches Interesse König Roberts an der Bibliothek, zahlreiche Schreiber werden gehalten, monatlich bezahlt, einige auf Staatskosten eingemiethet, was sie als Ausländer kennzeichnet; einige der Schreiber mögen zugleich Miniatoren gewesen $\operatorname{sein}^{1}$ ).

Unter anderen Erwerbungen finden sich auch zwei Psalter genannt, bei deren Bezahlung ausdrücklich Miniatur und Einband als einbezogen erwähnt werden, was auf frische Erledigung schliessen lässt ${ }^{2}$ ). Die Schreiber müssen, soweit wir ihre Namen kennen, überwiegend Italiener gewesen sein; Ausnahmen hievon bilden ein Gioranni de Ipra ${ }^{3}$ ), also, wie es scheint, ein Burgunder, und Roberto de Gondicur ${ }^{4}$ ), offenbar ein Franzose.

Für die an unserem Codex betheiligt gewesenen Künstler bestimmte Namen festzustellen, ist natürlich mit den heutigen Mitteln unmöglich: wir müssen uns begnügen, im Vorstehenden mit ziemlicher Gewissheit dargethan zu haben, dass wir es mit einem Denkmal zu thun haben, das der Kunstliebe des angiovinischen Hofes seine Entstehnng verdankt, jenes Hofes, der im 14. Jahrhundert durch die Heranziehung der nambaftesten Kunstkräfte Italiens annähernd jene geistige Einheit des Apenninenlandes herstellte, wie es über ein Jahrhundert später den Päpsten gelungen ist ${ }^{5}$ ).

Es erübrigt uns nur noch, die Schicksale des Buches an der Hand der spärlich gebotenen Behelfe nach Möglichkeit weiter zu verfolgen. Es wurde bereits mehrfach angedeutet, dass gewisse im Kalendar von späterer Hand nachgetragene Heiligennamen auf den Norden, nach

1) Arch. nap. 7, $216 \mathrm{zum}$ Jabre 1310, Re Roberto faceva copiare l'opera sua .... dal chierico Stefano... e dall altro chierico Errico il libro ... guesti chierici Roberto li teneva salariati a mese per trascrivere i libri della sua bibliotheca. Ebenda 221 ... fece scrivere ... dagli scrittori E. e St. predetto ... i libri ... che fece alluminare da' suoi miniatori. Dagegen scheinen Schreiber und Illuminator identisch im Regest 7, 246 (zum J. 1816): R. faceva scrivere miniare e legare libri per la sua biblioteca da fra Giovanni de Exarcellis mastro della capella regia ed eletto vescovo di Acerra, vgl. weiter $7,228,638 ; 8,28,25$ (zu 1s35), 26, 29 (zu 13s6), 210 (zu 1388), 226 (zu 1540).

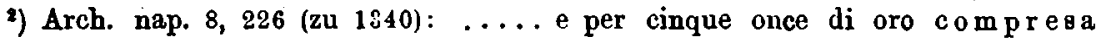
la miniatura e la legatura compro due Salterii, tre Antifonari, il testo degli Evangeli, l'uffizio del corpo di Cristo, due libri degli uffici comuni della Legenda de' Santi, ed altri libri... 8) Arch. 7, 228 zu 1319. 4) Arch. 8, 25 zu 1335. 5) Arch. 7, 363 sagt Faraglia von der Zeit der ersten Angiovinen : [Napoli era il centro d'Italia più potente e più ricco; in questo dunque convenivano i mercanti, gli artisti, i poeti: Giotto, Cino da Siena, Montano d'Arezzo, Pazzo e. Giovanni da Firenze, Boccaccio e Petrarca, Cavallino, Giacomo Gottifredo, Guglielmo di Verdalay e Miletto d'Auxerre. 
Denis angeblich auf Schweden hinweisen. Es sind im Ganzen 11 Namen, worunter allerdings 6 schwedische (wozu noch je eine translatio und canonisatio kommt); ferner je ein norwegischer (Olaf) und deutscher (Ansgar), zwei englische (Botulf Abt von Ikanhoe und Thonas von Canterbury), endlich ein dänischer (Kanut). Die schwedischen sind: Bischof Heinrich von Upsala, der Finnenapostel, Bischof Siegfried von Wexiö, Bischof Eskill von Strengen, die Witwe Helena, König Erich (mit seiner Translatio) und die schwedische Brigitta oder Birgitta (mit ihrer Canonisatio). Da die letztere am 7. Oct. 1391 durch Papst Bonifaz JX. canonisirt wurde ${ }^{1}$ ), ist zugleich eine Zeitgrenze für die Eintragung nach rückwärts gewonnen. Der Schriftcharakter lässt nicht weit unter dieses Jahr herabgehen. Dass der damalige Besitzer oder der Schreiber der Zusätze ein Schwede gewesen sein müsse, glaube ich nicht so unbedingt annehmen $\%$ müssen. Anskar, Kanut, Olaf und Birgitta genossen im Norden allgemeine Verehrung, wie sie sich beispielsweise auch in dem im Neukloster zu Wiener-Neustadt bewahrten Kalendar aus Brodersholm in Holstein neben anderen norddeutschen Heiligen finden ${ }^{2}$ ). Ausser den nordischen Heiligennamen finden sich auch altchristliche und andere beigesetzt, worunter Benedict v. Nursia, Antonius abbas und Bernhard v. Clairvaux, woraus man auf einen mönchischen Schreiber schliessen könnte; die Angabe der Feste des h. Dominicus und Petrus Martyr könnten hinwiederum auf einen Angehörigen des Predigerordens hinweisen.

Sehen wir uns nach den Verbindungen des angiovinischen Hauses mit dem Norden um, so begegnet uns zunächst Margarita, eine Tochter Philipps von Tarent, die mit König Eduard ron Schottland ( $†$ 1342) vermählt ward. Wenn wir uns erinnern, dass der Codex noch einige Schlussfolien enthält, die in der zweiten Hälfte des 14. Jahrh. geschrieben sein müssen, aber noch immer in den Ornamenten die angiovinischen Lilien aufweisen, so fällt jene Heirath wol ausser Betracht. Mehr Wahrscheinlichkeit hätte eine Conjectur, welche die am 15. Sept. 1376 vollzogene Vermählung Johanna's I. von Neapel mit Otto v. Braunschweig ( $\dagger$ Ende 1498) mit der Debertragung des Buches nach dem Norden in Verbindung brachte. Doch bin ich ebensowenig im Stande hiefür zwingende Belege beizubringen, als Denis für seine Erklärung, wie das Buch aus dem Norden nach Tirol gelangt sei. Die Hypothese, welche die Königin Christine von Schweden zur ehemaligen Eigenthümerin und Schenkerin des Buches an ein Nonnenkloster in TirolischHall macht, hat unstreitig viel Ansprechendes ${ }^{3}$ ). Diese wie viele ähn-

1) Rietz Scr. rer. suec. 3, 241 ft. Historia canonisationis sancte Birgitte. 2) Anzeiger f. Kunde d. deutsch. Vorzeit 2, 6. 9) Allerdings verliert gie durch 
liche Fragen müssen aber insolange unerledigt bleiben, als der äusserst fühlbare Mangel einer Geschichte unserer Hofbibliothek nicht beseitigt ist.

das jüngst erschienene Büchlein von A. Busson, Christine von Schweden in Tirol, viel an ihrer Glaubwürdigkeit. Die Exkönigin hat Hall weder auf der Reise berührt, noch von Innsbruck aus besucht. Von den Gästen hat, soweit die Nach. richten vorliegen, bloss der päpstliche Internuntins Holstenius Hall besucht, , am dort den Heilthumsschatz in dem königlichen Stift und die Salzpfanne zu besichtigen" (S. 68). Immerhin bleibt es möglich, dass Jemand ans dem zahlreichen Gefolge der Königin während des achttägigen Aufenthalts in Innsbruck (31. Oct. bis 8 . Nov. 1655) das nachbarliche Hall besucht hat. 